\title{
Synthesis of 1-alkyl triazolium triflate room temperature ionic liquids and their catalytic studies in multi-component Biginelli reaction
}

\author{
SANKARANARAYANAN NAGARAJAN, TANVEER M SHAIKH and \\ ELANGO KANDASAMY* \\ Department of Chemistry, Vel Tech University, Chennai 600 062, India \\ e-mail: elangoomc@gmail.com
}

MS received 24 January 2015; revised 25 April 2015; accepted 25 May 2015

\begin{abstract}
Synthesis of three Brønsted acid-based ionic liquids, namely, 1-ethyl-1,2,4-triazolium triflate (1a), 1-propyl-1,2,4-triazolium triflate (1b) and 1-butyl-1,2,4-triazolium triflate (1c), is described. These ionic liquids have been employed as catalysts for convenient and high-yielding one-pot synthesis of 3,4-dihydropyrimidin$2(1 \mathrm{H})$-ones and 3,4-dihydropyrimidin-2(1H)-thiones, which are Biginelli reaction products. Advantages of the methodology are operational convenience, short reaction times, avoidance of chromatographic purification and non-production of toxic waste. Further, the catalysts are easily recovered and reused without any noticeable diminution in their catalytic activity.
\end{abstract}

Keywords. Brønsted acid; ionic liquid; 1,2,4-Triazolium triflate; Biginelli reaction; Pyrimidones.

\section{Introduction}

The construction of C-C bond via multicomponent onepot reaction has given new pathway to a large variety of important compounds. ${ }^{1}$ Particularly, the Biginelli reaction is an example of such multicomponent bond forming reactions. ${ }^{2}$ Although this reaction was originally reported ${ }^{3}$ in 1893 using hydrochloric acid as catalyst, ${ }^{4}$ later several modifications have been reported in literature. Moreover, the Biginelli product, dihydropyrimidinone (DHPM) derivatives (figure 1) are associated with important bioactive properties, such as anti-cancer, anti-HIV, anti-hypertensive, anti-viral, calcium channel blockers, $\alpha$-1-antagonists and neuropeptide Y (NPY) antagonists, etc. ${ }^{5}$

In view of these useful properties, development of an environmentally benign and clean method is a concern both academia and industries. Since the discovery of this reaction, a variety of homogeneous and heterogeneous catalysts has been developed, which are effective in this one-pot transformation. Those methods involved use of a number of metal salts, such as $\mathrm{Li},{ }^{6} \mathrm{Fe},{ }^{7-10} \mathrm{Cu},{ }^{11,12} \mathrm{Ce},{ }^{13} \mathrm{Zr},{ }^{14} \mathrm{In},{ }^{16} \mathrm{Bi},{ }^{17} \mathrm{Yb},{ }^{18}$ $\mathrm{La},{ }^{19} \mathrm{Al},{ }^{20,21} \mathrm{Sn},{ }^{22} \mathrm{Mn},{ }^{23} \mathrm{Ti}^{24}$ and nanomaterials of $\mathrm{Fe}_{3} \mathrm{O}_{4} \cdot{ }^{25}$ Several zeolite catalyzed ${ }^{26-28}$ and non-metal acid catalyzed ${ }^{29-32}$ syntheses of dihydropyrimidinone have also been reported. However, some of these

\footnotetext{
*For correspondence
}

procedures are associated with certain limitations such as use of metal catalysts, expensive reagents, and drastic reaction conditions, use of microwave or ultrasonication which often resulted in unsatisfactory yields of product. On the other hand, employing metal catalysts also resulted in over-oxidized product and substantial amount of metal-waste, which are major problems towards a sustainable process. Therefore, the development of a new protocol toward this direction is an active area of research. In this context, several new reagents have been developed employing various ionic liquids as catalyst to carry out Biginelli reaction. ${ }^{33-37}$ Particularly, ionic liquids such as $\left[\mathrm{cmmim}\left[\left[\mathrm{BF}_{4}\right],{ }^{38} \mathrm{TMGT},{ }^{39}\right.\right.$ tri-(2-hydroxyethylammoniumacetate), ${ }^{40}[\mathrm{Gly}] \mathrm{NO}_{3},{ }^{41}$ $\mathrm{Hmim}\left[\mathrm{HSO}_{4}\right]^{42} \mathrm{Si}$ - $[\mathrm{SbSipim}]\left[\mathrm{PF}_{6}\right],{ }^{43}[$ bmim $]\left[\mathrm{MesO}_{4}\right]^{44}$ and $[\mathrm{BMIM}] \mathrm{OH}^{45}$ have been known to achieve this one-pot transformation.

We are interested in syntheses of a new class of ionic liquids and development of eco-friendly and reusable catalytic transformations. ${ }^{46}$ Recently, we have demonstrated Brønsted acid-based ionic liquids, 1,2,4triazolium methanesulfonate (figure 2) as catalyst in multicomponent Mannich reaction. ${ }^{47}$ In continuation of our efforts towards the development of sustainable process, we herein disclose an efficient and reusable protocol using 1,2,4-triazolium triflate Brønsted acidbased ionic liquids for the one-pot preparation of 3,4-dihydropyrimidin-2(1H)-ones and 3,4-dihydropyrimidin-2(1H)-thiones. 
<smiles>CCOC(=O)C1=C(C)NC(=S)NC1c1cccc(O)c1</smiles>

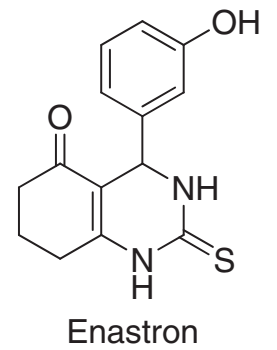<smiles>CC1=C(C(=O)OC(C)C)C(c2cccc([N+](=O)[O-])c2)N(C(N)=O)C(=S)N1</smiles>

SQ 32926,5

Figure 1. Dihydropyrimidinone derivatives.

\section{Experimental}

\subsection{Instruments}

${ }^{1} \mathrm{H}$ and ${ }^{13} \mathrm{C}$ NMR spectra were recorded on a Bruker Avance $400 \mathrm{MHz}$. Electrospray ionization mass spectrometry (ESI-MS) spectra were obtained with a Waters Q-TOF premier mass spectrometer.

\subsection{Materials}

Solvents were freshly distilled prior to use and glassware was dried in oven at $120^{\circ} \mathrm{C}$ overnight. Trifluromethanesulfonic acid was purchased from Sigma Aldrich. 1,2,4-triazole, ethyl bromide, propyl bromide and $n$-butyl bromide were purchased from SD fine chemicals, India. Starting materials such as urea, thiourea, ethylacetoacetate, methylacetoacetate, benzaldehyde, 4-methyl benzaldehyde, 4-isopropylbenzaldehyde, 4-flurobenzaldehyde, 4-bromobenzaldehyde, 4-chloro benzaldehyde, 3-hydroxybenzaldehyde were obtained from SRL, India. All the chemicals were used without further purification.

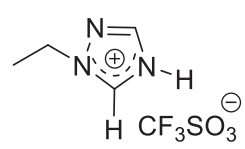

$1 \mathbf{a}$

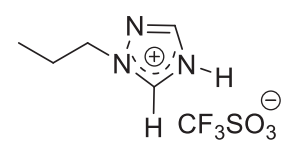

$1 \mathrm{~b}$

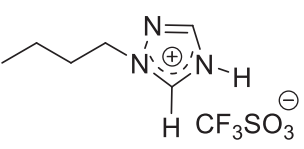

$1 c$
Figure 2. Bronsted acid-based ionic liquids: 1,2,4-triazolium triflates.

\subsection{Synthesis of 1-alkyl-1,2,4-triazolium triflate based RTILs (scheme 1)}

2.3a Common procedure for the synthesis of ionic liquids 1a-c: 1-Ethyl-1,2,4-triazolium triflate (1a), 1Propyl-1,2,4-triazolum triflate (1b) and 1-butyl-1,2,4triazolium triflate (1c). To a solution of 1-alkyl-1, 2, 4triazoles $(10 \mathrm{mmol})($ alkyl $=\mathrm{Et}, \mathrm{Pr}$ and $\mathrm{Bu})$ in toluene $(10 \mathrm{~mL})$ trifluromethanesulfonic acid $(10 \mathrm{mmol})$ was added drop wise. This reaction mixture was then heated to $80^{\circ} \mathrm{C}$ for $12 \mathrm{~h}$. After completion of the reaction, the flask was cooled to room temperature $\left(25^{\circ} \mathrm{C}\right)$ and excess of toluene was removed under reduced pressure. The resulting residue was thoroughly washed with hexane $(20 \mathrm{~mL} \times 2)$ and further dried over vacuum to afford pure catalyst 1a-c.

\subsection{General Procedure for the preparation of 3,4-dihydropyrimidin-2(1H)-Ones/thiones}

Catalyst 1a-c $(10 \mathrm{~mol} \%)$ was added to a solution of aldehyde $(1.0 \mathrm{mmol}), \beta$-ketoester $(1.5 \mathrm{mmol})$ and urea or thiourea $(2.0 \mathrm{mmol})$ in ethanol $(0.5 \mathrm{~mL})$. The reaction mixture was heated at $80^{\circ} \mathrm{C}$ using oil bath for the specified time $(0-5 \mathrm{~h})$. The progress of the reaction was monitored by TLC. After completion, the reaction mixture was cooled to room temperature and subsequently quenched with a mixture of water:ethanol $(5: 0.5 \mathrm{~mL})$. The solid product was filtered and washed with $n$-hexane $(5 \mathrm{~mL} \times$ $2)$, which afforded pure 3,4-dihydropyrimidin-2(1H)ones or 3,4-dihydropyrimidin-2(1H)-thiones in pure form.

\subsection{Catalyst recycling study}

The catalyst was separated from the reaction mixture by simple filtration technique. Then the filtrate was concentrated under reduced pressure to remove excess ethanol and water. Then the residue was washed with $5 \mathrm{~mL}$ of hexane:ethyl acetate $(4: 1)$ and dried over vacuum for $1 \mathrm{~h}$, which was directly used in reusability studies.

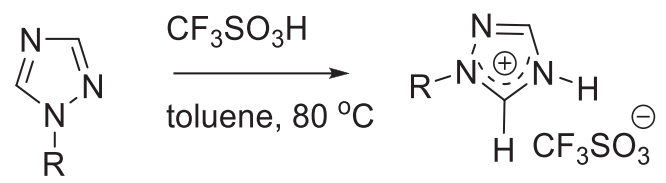

Scheme 1. Synthesis of 1-alkyl-1,2,4-triazolium triflate based RTILs. 


\subsection{Spectral data for selected compounds}

2.6a 1-ethyl-1,2,4-triazolium triflate (1a): Colorless liquid: yield $85 \% ;{ }^{1} \mathrm{H} \mathrm{NMR}\left(\mathrm{CDCl}_{3}, 400 \mathrm{MHz}\right): \delta 11.9$ $(\mathrm{s}, 1 \mathrm{H}, 4-\mathrm{NH}), 9.6(\mathrm{~s}, 1 \mathrm{H}, 5-\mathrm{CH}), 8.6(\mathrm{~s}, 1 \mathrm{H}, 3-$ $\mathrm{CH}) 4.5(\mathrm{q}, 2 \mathrm{H}), 1.5(\mathrm{t}, 3 \mathrm{H}) ;{ }^{13} \mathrm{C} \mathrm{NMR}\left(\mathrm{CDCl}_{3}\right.$, $100 \mathrm{MHz}$ ): $\delta 143.3$ (C-5), 140.5 (C-3), 124.8,121.6, 118.4,115.3 $\left(\mathrm{CF}_{3}-\mathrm{SO}_{3}\right) 47.4,13.7 ;{ }^{19} \mathrm{~F}$ NMR (DMSOd6): $-78.42 \mathrm{ppm}$; ES-MS m/z: 98.0711 [M- $\mathrm{CF}_{3}-$ $\left.\mathrm{SO}_{3}\right]^{+}$.

2.6b 1-Propyl 1,2,4-triazolium triflate ( $1 \mathrm{~b})$ : Colorless liquid, yield 94\%; ${ }^{1} \mathrm{H} \mathrm{NMR}\left(\mathrm{CDCl}_{3}, 300 \mathrm{MHz}\right)$ : $\delta 11.9$ (s, 1H, NH), $9.6(\mathrm{~s}, 1 \mathrm{H}, 5-\mathrm{CH}), 8.5$ (s, 1H, 3$\mathrm{CH}), 4.3(\mathrm{t}, 2 \mathrm{H}), 1.9(\mathrm{~m}, 2 \mathrm{H}), 0.9(\mathrm{t}, 3 \mathrm{H}) ;{ }^{13} \mathrm{C} \mathrm{NMR}$ $\left(\mathrm{CDCl}_{3}, 75 \mathrm{MHz}\right): \delta 143.6(\mathrm{C}-5), 141.1$ (C-3), 122.3, $118.0\left(\mathrm{CF}_{3}-\mathrm{SO}_{3}\right)$ 53.6, 22.3, 10.4; ${ }^{19} \mathrm{~F} \mathrm{NMR}\left(\mathrm{CDCl}_{3}\right)$ : -78.95 ppm; ES-MS m/z: 112.0869. [M- $\left.\mathrm{CF}_{3}-\mathrm{SO}_{3}\right]^{+}$.

2.6c 1-butyl-1,2,4-triazolium triflate (1c): Colorless liquid: yield 95\%; ${ }^{1} \mathrm{H} \mathrm{NMR}\left(\mathrm{CDCl}_{3}, 400 \mathrm{MHz}\right): \delta 11.7$ (s, 1H, 4-NH ), 9.5 (s, 1H, 5-CH ), $8.6(\mathrm{~s}, 1 \mathrm{H}, 3-\mathrm{CH}) 4.4$ (t ,2H ) $1.9(\mathrm{~m}, 2 \mathrm{H}) 1.4(\mathrm{~m}, 2 \mathrm{H}) 1.3(\mathrm{t}, 3 \mathrm{H}) ;{ }^{13} \mathrm{C} \mathrm{NMR}$ $\left(\mathrm{CDCl}_{3}, 100 \mathrm{MHz}\right): \delta 144.0(\mathrm{C}-5), 144.1$ (C-3), 124.8, 121.7, 118.5, $115.3\left(\mathrm{CF}_{3}-\mathrm{SO}_{3}\right), 51.5,30.7,19.1,13.0$; ${ }^{19} \mathrm{~F}$ NMR (DMSO- $d_{6}$ ): -77.91; ES-MS m/z:126.0963 $\left[\mathrm{M}-\mathrm{CF}_{3}-\mathrm{SO}_{3}\right]^{+}$.

2.6d 5-Methoxycarbonyl-6-methyl-4-phenyl-3,4-dihydropyrimidin-2(1H)-one: (table 4, entry 1$){ }^{1} \mathrm{HNMR}$ $\left(\mathrm{CDCl}_{3}, 400 \mathrm{MHz}\right): \delta 8.3(\mathrm{~s}, 1 \mathrm{H}), 7.3(\mathrm{~m}, 5 \mathrm{H}), 5.9$ $(\mathrm{s}, 1 \mathrm{H}), 5.3(\mathrm{~d}, J=4.0 \mathrm{~Hz}, 1 \mathrm{H}), 3.6(\mathrm{~s}, 3 \mathrm{H}), 2.3(\mathrm{~s}$, $3 \mathrm{H}) ;{ }^{13} \mathrm{CNMR}\left(\mathrm{CDCl}_{3}, 100 \mathrm{MHz},\right): \delta \quad 166.1,153.5$, $146.6,143.6,128.7,127.9,126.5,101.1,55.5,51.1$, 18.7.

2.6e 5-methoxycarbonyl-4(4-isopropylphenyl)-6-methyl-3,4-dihydropyrimidin-2(1H)-one: (table 4, entry 3): M.p.: $178-179^{\circ} \mathrm{C} ;{ }^{1} \mathrm{HNMR}\left(\mathrm{CDCl}_{3}, 400 \mathrm{MHz}\right): \delta$ $8.4(\mathrm{~s}, 1 \mathrm{H}), 7.2(\mathrm{~m}, 4 \mathrm{H}), 5.8(\mathrm{~s}, 1 \mathrm{H}), 5.3(\mathrm{~d}, J=4.0 \mathrm{~Hz}$, $1 \mathrm{H}), 3.6(\mathrm{~s}, 3 \mathrm{H}), 2.9(\mathrm{~m}, 1 \mathrm{H}), 2.3(\mathrm{~s}, 3 \mathrm{H}), 1.2(\mathrm{~d}$, $J=8.0 \mathrm{~Hz}, 6 \mathrm{H}) ;{ }^{13} \mathrm{CNMR}\left(\mathrm{CDCl}_{3}, 100 \mathrm{MHz}\right): \delta$ 166.2, 153.7, 148.5, 146.5, 141.0, 126.8, 126.4, 101.3, $55.2,51.16,33.7,23.9,18.6$; (LC-Mass, $\mathrm{m} / \mathrm{z})=289$ $\left(\mathrm{M}^{+}+1\right)$.

$2.6 f$ 5-Ethoxycarbonyl-4(3-hydroxyphenyl)-6-methyl3,4-dihydropyrimidin-2(1H)-one: (table 4, entry 16) ${ }^{1} \mathrm{HNMR}$ (DMSO- $d_{6}, 400 \mathrm{MHz}$ ): $\delta 9.3(\mathrm{~s}, 1 \mathrm{H}), 9.1(\mathrm{~d}$, $J=1.6 \mathrm{~Hz}, 1 \mathrm{H}), 7.6(\mathrm{~m}, 1 \mathrm{H}) 7.0(\mathrm{t}, J=8.0 \mathrm{~Hz}, 1 \mathrm{H})$, $6.6(\mathrm{~m}, 2 \mathrm{H}), 6.6(\mathrm{~m}, 1 \mathrm{H}), 5.0(\mathrm{~s}, 1 \mathrm{H}), 3.9(\mathrm{q}, 2 \mathrm{H}), 2.4$ $(\mathrm{m}, 3 \mathrm{H}), 1.0(\mathrm{t}, J=7.2 \mathrm{~Hz}, 3 \mathrm{H}) ;{ }^{13} \mathrm{CNMR}$ (DMSO$\left.d_{6}, 100 \mathrm{MHz},\right): \delta 165.4,157.3,152.2,148.1,146.2$, $129.3,116.9,114.19,113.1,99.4,59.2,53.8,17.7$, 14.1 .

2.6g 5-Methoxycarbonyl-6-methyl-4-phenyl-3,4-dihydropyrimidin-2(1H)-thione: (table 4, entry 17) ${ }^{1} \mathrm{HNMR}$ $\left(\mathrm{CDCl}_{3}, 400 \mathrm{MHz}\right): \delta 8.5(\mathrm{~s}, 1 \mathrm{H}), 7.2(\mathrm{~m}, 3 \mathrm{H}), 7.0$ $(\mathrm{m}, 2 \mathrm{H}), 6.1(\mathrm{~s}, 1 \mathrm{H}), 5.3(\mathrm{~d}, J=4.0 \mathrm{~Hz}, 1 \mathrm{H}), 3.6(\mathrm{~s}$, $3 \mathrm{H}), 2.3$ (s, 3H); ${ }^{13} \mathrm{CNMR}\left(\mathrm{CDCl}_{3}, 400 \mathrm{MHz}\right): \delta 166.0$, $163.5,161.1,153.6,146.7,139.5,139.5,128.2,128.1$, $115.7,115.5,101.1,54.8,51.2,18.6$.

2.6h 5-ethoxycarbonyl-4(3-hydroxyphenyl)-6-methyl3,4-dihydropyrimidin-2(1H)-thione: (table 4, entry 32) ${ }^{1} \mathrm{HNMR}$ (DMSO- $\left.d_{6}, 400 \mathrm{MHz}\right): \delta 10.2(\mathrm{~s}, 1 \mathrm{H})$, $9.5(\mathrm{~d}, 1 \mathrm{H}), 9.4(\mathrm{~s}, 1 \mathrm{H}), 7.1(\mathrm{~m}, 1 \mathrm{H}), 6.6(\mathrm{~d}, 3 \mathrm{H}), 5.0$ $(\mathrm{d}, 1 \mathrm{H}), 4.0(\mathrm{q}, 2 \mathrm{H}), 2.2(\mathrm{~s}, 3 \mathrm{H}), 1.1(\mathrm{t}, \mathrm{J}=7.2 \mathrm{~Hz})$; ${ }^{13}$ CNMR (DMSO- $d_{6}, 100 \mathrm{MHz}$ ): $\delta 174.1,165.1,157.4$, $144.8,144.7,129.4,117.0,114.6,113.2,100.7,59.5$, $53.9,17.1,14.0$.

\section{Results and Discussion}

Initially, we studied the suitable reaction conditions employing ionic liquids $\mathbf{1 a}-\mathbf{c}$ as the catalyst to promote one-pot Biginelli reaction using benzaldehyde, ethyl acetoactetae and urea with altered catalyst loading, and the results are summarized in table 1. It was observed that without catalyst the reaction failed to give any product (table 1, entry 1). While the reaction was performed in the presence of $5 \mathrm{~mol} \%$ ILs 1a-c, the desired product 5 was obtained in 74-79\% yield, respectively (entry 2). Indeed the yield of dihydropyrimidinone increased to 93-95\%, using 10 mol\% ILs 1a-c, with full conversion in a relatively shorter time of $20 \mathrm{~min}$ (entry 3 ). While increasing the catalyst loading the yield of $\mathbf{5}$ decreased. The reaction did not significantly improve because the ionic liquids are acidic and high catalyst loading leads to the formation of side products.

In order to determine the effect of solvents, we investigated the Biginelli reaction in different solvent systems and the results are presented in table 2. It was observed that in the absence of solvent (neat) dihydropyrimidinone was obtained in $75 \%$ yield (table 2 , entry 1). Among all the solvents screened, it was found that ethanol is the most suitable solvent for this reaction. However, in non-polar solvents such as toluene, tetrahydrofuran, acetonitrile and dichloromethane the reaction resulted in moderate yield, which might be due to poor solubility of starting materials. After extensive 
Table 1. Optimization of reaction conditions: role of catalyst for the synthesis of 3,4-dihydropyrimidin-2(1H)-one ${ }^{\mathrm{a}}$.

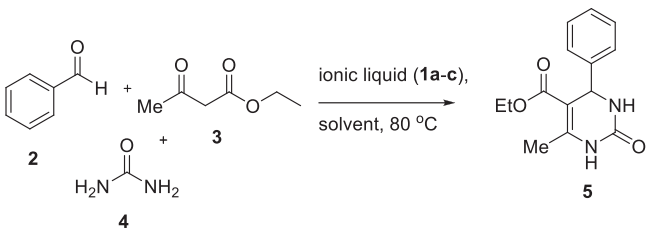

\begin{tabular}{|c|c|c|c|c|c|c|c|}
\hline \multirow[b]{2}{*}{ entry } & \multirow[b]{2}{*}{ RTILS mole (\%) } & \multicolumn{2}{|c|}{ TrEtHTA (1a) } & \multicolumn{2}{|c|}{ TrPrHTA (1b) } & \multicolumn{2}{|c|}{ TrBuHTA (1c) } \\
\hline & & time $(\mathrm{h})$ & yield $^{\mathrm{b}, \mathrm{c}}(\%)$ & time $(\mathrm{h})$ & yield $^{\mathrm{b}, \mathrm{c}}(\%)$ & time $(\mathrm{h})$ & yield $^{\mathrm{b}, \mathrm{c}}(\%)$ \\
\hline 1 & 0 & 1.0 & 0 & 1.0 & 0 & 1.0 & 0 \\
\hline 2 & 5 & 1:0 & 74 & $1: 0$ & 75 & $1: 0$ & 79 \\
\hline 3 & 10 & $0: 45$ & 93 & $0: 30$ & 93 & $0: 20$ & 95 \\
\hline 4 & 15 & $0: 35$ & 90 & $0: 30$ & 90 & $0: 30$ & 90 \\
\hline 5 & 20 & $0: 25$ & 84 & $0: 25$ & 84 & $0: 20$ & 85 \\
\hline
\end{tabular}

${ }^{a}$ Reaction conditions: benzaldehyde $(1.0 \mathrm{mmol})$, ethyl acetoacetate $(1.5 \mathrm{mmol})$, urea $(2.0 \mathrm{mmol})$ and RTILs (10 mol\%);

${ }^{b}$ isolated yield;

${ }^{c}$ products were characterized by M.p., ${ }^{1} \mathrm{H}$ and ${ }^{13} \mathrm{C}-\mathrm{NMR}$

Table 2. Optimization of reaction conditions: solvent study for the synthesis of 3,4dihydropyrimidin-2(1H)-one ${ }^{\mathrm{a}}$.

\begin{tabular}{|c|c|c|c|c|c|c|c|}
\hline \multirow[b]{2}{*}{ entry } & \multirow[b]{2}{*}{ solvent } & \multicolumn{2}{|c|}{ TrEtHTA (1a) } & \multicolumn{2}{|c|}{ TrPrHTA (1b) } & \multicolumn{2}{|c|}{ TrBuHTA (1c) } \\
\hline & & time (h) & yield $(\%)^{\mathrm{b}, \mathrm{c}}$ & time (h) & yield $(\%)^{\mathrm{b}, \mathrm{c}}$ & time $(\mathrm{h})$ & yield $(\%)^{b, c}$ \\
\hline 1 & neat & 1.0 & 81 & 1.0 & 82 & 1.0 & 75 \\
\hline 2 & water & 1.0 & 54 & 1.0 & 54 & 1.0 & 65 \\
\hline 3 & methanol & 1.0 & 60 & 1.0 & 60 & 1.0 & 62 \\
\hline 4 & ethanol & 0.45 & 93 & $0: 30$ & 93 & 0.20 & 95 \\
\hline 5 & dichloromethane & 1.0 & 45 & 1.0 & 50 & 1.0 & 51 \\
\hline 6 & toluene & 1.0 & 0 & 1.0 & 0 & 1.0 & 0 \\
\hline 7 & tetrahydrofuran & 1.0 & 34 & 1.0 & 45 & 1.0 & 50 \\
\hline 8 & acetonitrille & 1.0 & 55 & 1.0 & 56 & 1.0 & 56 \\
\hline
\end{tabular}

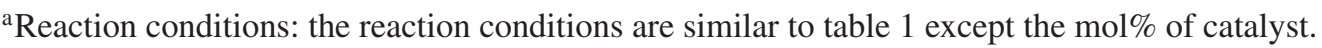

Table 3. Catalyst reusability study in three component Biginelli reaction ${ }^{\mathrm{a}}$.

\begin{tabular}{|c|c|c|c|c|c|c|c|}
\hline \multirow[b]{2}{*}{ entry } & \multirow[b]{2}{*}{ cycle } & \multicolumn{2}{|c|}{ TrEtHTA (1a) } & \multicolumn{2}{|c|}{ TrPrHTA (1b) } & \multicolumn{2}{|c|}{ TrBuHTA (1c) } \\
\hline & & time $(\mathrm{h})$ & yield (\%) & time (h) & yield (\%) & time (h) & yield (\%) \\
\hline 1 & 0 & $0: 45$ & 93 & $0: 30$ & 93 & $0: 20$ & 95 \\
\hline 2 & 1 & $1: 0$ & 90 & $0: 45$ & 91 & $0: 30$ & 92 \\
\hline 3 & 2 & $1: 0$ & 89 & $1: 0$ & 91 & $0: 30$ & 90 \\
\hline 4 & 3 & 1:0 & 89 & $1: 0$ & 90 & $0: 45$ & 90 \\
\hline
\end{tabular}

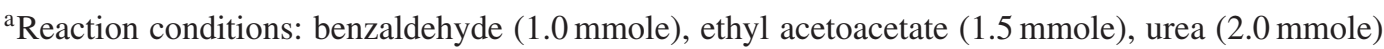
and RTILs (10 mol\%), EtOH $(1 \mathrm{~mL}), 80^{\circ} \mathrm{C}$.

screening of different reaction parameters, the optimized reaction conditions involved aldehyde $(1.0 \mathrm{mmol}), \beta$ ketoesters $(1.5 \mathrm{mmol})$, urea or thiourea $(2.0 \mathrm{mmol})$ and ionic liquids $\mathbf{1 a}$ or $\mathbf{1 b}$ or $\mathbf{1 c}(10 \mathrm{~mol} \%)$ in ethanol as solvent at $80^{\circ} \mathrm{C}$ to provide the desired product 5 in excellent yield.

We also investigated the reusability of the catalyst 1a-c and the results are described in table 3 . 
Table 4. Substrate scope for the Biginelli reaction for the synthesis of 3, 4-dihydropyrimidin-2(1H)-ones and thiones ${ }^{\mathrm{a}}$.<smiles>[R3]COC(=O)CC(C)=O</smiles>

\begin{tabular}{|c|c|c|c|c|c|c|c|c|c|c|c|}
\hline \multirow[b]{3}{*}{ entry } & \multirow[b]{3}{*}{$\mathrm{R}_{1}$} & \multirow[b]{3}{*}{$\mathrm{R}_{2}$} & \multirow[b]{3}{*}{$\mathrm{R}_{3}$} & \multirow[b]{3}{*}{$\mathrm{X}$} & \multicolumn{6}{|c|}{ RTILs } & \multirow[b]{3}{*}{ M.p. ${ }^{(c)}$} \\
\hline & & & & & \multicolumn{2}{|c|}{$1 \mathrm{a}$} & \multicolumn{2}{|c|}{ 1b } & \multicolumn{2}{|c|}{$1 c$} & \\
\hline & & & & & time (h) & yield $^{\mathrm{b}}(\%)$ & time (h) & yield $^{\mathrm{b}}(\%)$ & time (h) & yield $^{\mathrm{b}}(\%)$ & \\
\hline 1 & $\mathrm{H}$ & $\mathrm{H}$ & $\mathrm{H}$ & $\mathrm{O}$ & 1.0 & 93 & $0: 40$ & 94 & 0.30 & 95 & $209-210^{48}$ \\
\hline 2 & $4-\mathrm{CH}_{3}$ & $\mathrm{H}$ & $\mathrm{H}$ & $\mathrm{O}$ & 1.30 & 84 & $1: 30$ & 83 & 1.0 & 87 & $207-208^{49}$ \\
\hline 3 & $4-{ }^{\mathrm{i}} \mathrm{Pr}$ & $\mathrm{H}$ & $\mathrm{H}$ & $\mathrm{O}$ & 2.0 & 86 & $2: 0$ & 88 & 1.3 & 89 & $178-179$ \\
\hline 4 & $4-\mathrm{OCH}_{3}$ & $\mathrm{H}$ & $\mathrm{H}$ & $\mathrm{O}$ & 3.0 & 88 & $1: 30$ & 91 & 1.0 & 91 & $190-191^{48}$ \\
\hline 5 & $4-\mathrm{F}$ & $\mathrm{H}$ & $\mathrm{H}$ & $\mathrm{O}$ & 3.30 & 76 & $3: 30$ & 70 & 3.0 & 75 & $191-192^{50}$ \\
\hline 6 & $4-\mathrm{Cl}$ & $\mathrm{H}$ & $\mathrm{H}$ & $\mathrm{O}$ & 4.0 & 88 & $3: 30$ & 87 & 3.0 & 89 & $204-205^{48}$ \\
\hline 7 & $4-\mathrm{Br}$ & $\mathrm{H}$ & $\mathrm{H}$ & $\mathrm{O}$ & 3.30 & 80 & $3: 30$ & 82 & 3.3 & 81 & $218-219^{56}$ \\
\hline 8 & $\mathrm{H}$ & $3-\mathrm{OH}$ & $\mathrm{H}$ & $\mathrm{O}$ & 1.0 & 75 & $0: 50$ & 75 & 0.4 & 77 & $221-222^{52}$ \\
\hline 9 & $\mathrm{H}$ & $\mathrm{H}$ & $\mathrm{CH}_{3}$ & $\mathrm{O}$ & 0.45 & 93 & $0: 30$ & 93 & 0.2 & 95 & $200-201^{48}$ \\
\hline 10 & $4-\mathrm{CH}_{3}$ & $\mathrm{H}$ & $\mathrm{CH}_{3}$ & $\mathrm{O}$ & 2.0 & 87 & $2: 0$ & 87 & 2.0 & 89 & $212-214^{50}$ \\
\hline 11 & $4-{ }^{i} \mathrm{Pr}$ & $\mathrm{H}$ & $\mathrm{CH}_{3}$ & $\mathrm{O}$ & 4.0 & 85 & 4:0 & 86 & 3.3 & 89 & $140-141^{51}$ \\
\hline 12 & $4-\mathrm{OCH}_{3}$ & $\mathrm{H}$ & $\mathrm{CH}_{3}$ & $\mathrm{O}$ & 3.0 & 90 & $3: 0$ & 90 & 2.3 & 93 & $200-201^{48}$ \\
\hline 13 & $4-\mathrm{F}$ & $\mathrm{H}$ & $\mathrm{CH}_{3}$ & $\mathrm{O}$ & 4.0 & 77 & $4: 0$ & 75 & 3.0 & 78 & $173-174^{42}$ \\
\hline 14 & $4-\mathrm{Cl}$ & $\mathrm{H}$ & $\mathrm{CH}_{3}$ & $\mathrm{O}$ & 2.0 & 80 & $3: 30$ & 84 & $3: 0$ & 87 & $215-216^{48}$ \\
\hline 15 & $4-\mathrm{Br}$ & $\mathrm{H}$ & $\mathrm{CH}_{3}$ & $\mathrm{O}$ & 3.0 & 72 & $3: 0$ & 75 & 2.3 & 76 & $214-215^{50}$ \\
\hline 16 & $\mathrm{H}$ & $3-\mathrm{OH}$ & $\mathrm{CH}_{3}$ & $\mathrm{O}$ & 1.0 & 70 & $0: 45$ & 70 & 0.30 & 74 & $164-165^{51}$ \\
\hline 17 & $\mathrm{H}$ & $\mathrm{H}$ & $\mathrm{H}$ & $\mathrm{S}$ & 1.30 & 90 & 1:0 & 90 & 0.45 & 91 & $220-222^{48}$ \\
\hline 18 & 4- $\mathrm{CH}_{3}$ & $\mathrm{H}$ & $\mathrm{H}$ & S & 2.0 & 85 & $2: 0$ & 88 & 2.0 & 89 & $154-155^{49}$ \\
\hline 19 & $4{ }^{\mathrm{i}} \mathrm{Pr}$ & $\mathrm{H}$ & $\mathrm{H}$ & $S$ & 3.0 & 86 & $3: 0$ & 86 & 2.30 & 88 & $169-171^{55}$ \\
\hline 20 & $4-\mathrm{OCH}_{3}$ & $\mathrm{H}$ & $\mathrm{H}$ & S & 2.30 & 83 & $2: 30$ & 80 & 3.0 & 81 & $177-178^{48}$ \\
\hline 21 & 4-F & $\mathrm{H}$ & $\mathrm{H}$ & S & 3.30 & 76 & $3: 0$ & 75 & 3.0 & 76 & $183-184^{48}$ \\
\hline 22 & $4-\mathrm{Cl}$ & $\mathrm{H}$ & $\mathrm{H}$ & $\mathrm{S}$ & 5.0 & 84 & $4: 30$ & 87 & 4.0 & 88 & $152-153^{48}$ \\
\hline 23 & $4-\mathrm{Br}$ & $\mathrm{H}$ & $\mathrm{H}$ & S & 4.0 & 76 & $4: 0$ & 75 & 3.30 & 78 & $178-179^{48}$ \\
\hline 24 & $\mathrm{H}$ & $3-\mathrm{OH}$ & $\mathrm{H}$ & $S$ & 3.30 & 72 & $3: 0$ & 70 & 3.0 & 74 & $207-209^{53}$ \\
\hline 25 & $\mathrm{H}$ & $\mathrm{H}$ & $\mathrm{CH}_{3}$ & $S$ & 2.0 & 84 & $2: 0$ & 87 & 1.30 & 89 & $204-209^{48}$ \\
\hline 26 & $4-\mathrm{CH}_{3}$ & $\mathrm{H}$ & $\mathrm{CH}_{3}$ & S & 3.30 & 83 & $3: 30$ & 83 & 3.0 & 83 & $191-193^{49}$ \\
\hline 27 & $4-^{\mathrm{i}} \mathrm{Pr}$ & $\mathrm{H}$ & $\mathrm{CH}_{3}$ & $\mathrm{~S}$ & 3.30 & 87 & $3: 0$ & 89 & 2.30 & 90 & $138-140^{54}$ \\
\hline 28 & $4-\mathrm{OCH}_{3}$ & $\mathrm{H}$ & $\mathrm{CH}_{3}$ & $\mathrm{~S}$ & 3.0 & 90 & $3: 0$ & 92 & 2.0 & 94 & $154-155^{48}$ \\
\hline 29 & 4-F & $\mathrm{H}$ & $\mathrm{CH}_{3}$ & $\mathrm{~S}$ & 4.30 & 69 & $4: 30$ & 74 & 3.0 & 75 & $186-187^{48}$ \\
\hline 30 & $4-\mathrm{Cl}$ & $\mathrm{H}$ & $\mathrm{CH}_{3}$ & $\mathrm{~S}$ & 2.30 & 79 & $2: 30$ & 82 & 2.0 & 84 & $192-193^{48}$ \\
\hline 31 & $4-\mathrm{Br}$ & $\mathrm{H}$ & $\mathrm{CH}_{3}$ & S & 3.0 & 70 & $2: 30$ & 73 & 3.0 & 75 & $190-191^{48}$ \\
\hline 32 & $\mathrm{H}$ & $3-\mathrm{OH}$ & $\mathrm{CH}_{3}$ & $\mathrm{~S}$ & 1.30 & 69 & $1: 30$ & 70 & 1.0 & 72 & $182-184^{51}$ \\
\hline
\end{tabular}

${ }^{a}$ Reaction conditions: benzaldehyde $(1.0 \mathrm{mmol})$, ethyl acetoacetate $(1.5 \mathrm{mmol})$, urea $(2.0 \mathrm{mmol})$ and RTILs $(10 \mathrm{~mol} \%)$; ${ }^{b}$ isolated yield; ${ }^{c}$ products were characterized by M.p., ${ }^{1} \mathrm{H}$ and ${ }^{13} \mathrm{C}-\mathrm{NMR}$

The catalyst was recovered from the reaction mixture by using simple filtration technique. The filtrate was dried over vacuum to remove excess ethanol. Then the crude residue was washed with mixture of solvents, hexane:ethyl acetate (4:1), subsequently dried over vacuum for $30 \mathrm{~min}$. This catalyst was directly subjected to Biginelli reaction using the model reaction between benzaldehyde, methyl acetoacetate and urea with our optimized reaction conditions. It is important to note that the recycled catalysts $(\mathbf{1 a}-\mathbf{c})$ produced excellent yields of dihydropyrimidinone (5) in 90-95\%, respectively (table 3 , entries 1-4). It was observed that the yields were consistent without significant loss in its catalytic activity. 
With these optimized conditions in hand, we extended the scope of this methodology in synthesizing various 3,4-dihydropyrimidin-2(1H)-ones and 3,4-dihydropyrimidin-2(1H)-thiones, Biginelli products and the results are summarized in table 4. The reaction of 4-methyl benzaldehyde proceeded to give the desired products methyl substituted 3,4dihydropyrimidin-2-ones (table 4, entry 2) and 3,4dihydropyrimidin-2-thiones (table 4, entry 18 ) in excellent isolated yields. The effect of several substituents on benzaldehyde, such as alkyl, alkoxy, halides, F, $\mathrm{Cl}$ and $\mathrm{Br}$ were investigated, which smoothly resulted to the formation of products $\mathbf{5}$ in excellent yields. Similarly, the $\beta$-keto ester substituted with methylor ethyl-acetoacetate did not alter the yield of Biginelli product (entries 1 to 7 and 25 to 30). Interestingly, it was found that the reaction in ionic liquid 1c was faster compared with ionic liquids $\mathbf{1 a}$ and $\mathbf{1 b}$. Probably, the substituents in ionic liquids have significant steric effects which impact on the rate of the reaction.

\section{Conclusions}

In summary, we have developed a convenient, sustainable and reusable protocol for the multicomponent Biginelli reaction, using 1,2,4-triazolium triflate based ionic liquids as catalyst. The Biginelli products 3,4-dihydropyrimidin-2(1H)-ones and 3,4dihydropyrimidin-2 $(1 \mathrm{H})$-thiones have been obtained in excellent isolated yields. The product was isolated by simple filtration technique without chromatographic purification. It is important to note that the catalysts were recovered and reused without loss of its catalytic activity. This protocol involves inexpensive reagents and reusable catalyst which may be suitable for largescale preparation of important heterocycles in academia and industries.

\section{Supplementary Information}

Complete experimental procedure for synthesis of ionic liquids 1a-c and the corresponding scanned spectra, ${ }^{1} \mathrm{H}-,{ }^{13} \mathrm{C}-,{ }^{19} \mathrm{~F}-\mathrm{NMR}$, ESI-MS, LC-MS are given in Supplementary Information as figures $\mathrm{S} 2$ to S13, respectively. Also, synthetic procedure to the preparation of 3,4-dihydropyrimidin-2(1H)-ones and 3,4-dihydropyrimidin-2(1H)-thiones along with some selected scanned spectra of compounds in table 4 , entries 1,3,16,17 and 32 are also given (figure S14 to S24). Supplementary Information is available at www. ias.ac.in/chemsci.

\section{Acknowledgements}

The authors thank the Department of Science and Technology, Science and Engineering Research Board (DST-SERB, SR/FT/CS-60/2011), India for providing research fund. Also the authors thank Dr. G. Anantharaman Department of Chemistry, India Institute of Technology Kanpur for NMR and ESI-MS measurements.

\section{References}

1. Posner G H 1986 Chem. Rev. 86831

2. Safari J and Ravandi S G 2014 J. Mol. Struct. 1065241

3. Biginelli P 1893 Gazz. Chim. Ital. 23360

4. Alvim H G O, Lima T B, Oliveira A L, Oliveira H C B, Silva F M, Gozzo F C, Souza R Y, Silva W A and Net B A D 2014 J. Org. Chem. 793383

5. Murata H Ishitani H and Iwamoto M 2010 Org. Biomol. Chem. 81202 and the references cited therein

6. Rudrawa S 2005 Synlett. 71197

7. Cepanec I, Litvic M, Bartolincic A and Lovric M 2005 Tetrahedron 614275

8. Adibi H, Samimi H A and Beygzadeh M 2007 Catal. Commun. 82119

9. Mondal J, Sen T and Bhaumik A 2012 Dalton Trans. 41 6173

10. Seyedi N 2013 Transition. Met. Chem. 3893

11. Paraskar A S, Dewkar G K and Sudalai A 2003 Tetrahedron Lett. $\mathbf{4 4} 3305$

12. Reddy V Y, Kurva S and Tammishetti S 2004 Catal. Commun. 5511

13. Bose D S, Fatima L and Mereyala M H B 2003 J. Org. Chem. $\mathbf{6 8} 587$

14. Khaleghi S, Heravi M M, Khosroshahi M, Behbahani F K and Daroogheha Z 2008 Green Chem. Lett. Rev. 2 133

15. Reddy C V, Mahesh M, Raju P V K, Babu T R and Reddy V V N 2002 Tetrahedron Lett. 432657

16. Ranu B C, Hajra A and Jana U 2000 J. Org. Chem. 65 6270

17. Antoniotti S 2003 Synlett 101566

18. Zhang H, Zhou Z, Yao Z, Xu F and Shen Q 2009 Tetrahedron Lett. 501622

19. Lannou M I, Helion F and Namy J L 2008 Synlett 1105

20. Azizian J, Mohammadi A A, Karimi A R and Mohammadizadeh M R 2006 Applied Catal. A 30085

21. Hashem S 2009 Synth. Commun. 39958

22. Singh M O and Devi N S 2009 J. Org. Chem. 743141

23. Kumar K A, Kasthuraiah M, Reddy C S and Reddy C D 2001 Tetrahedron Lett. 427873

24. Safari J and Gandomi-Ravandi S 2014 New J. Chem. 38 3514

25. Zamani F and Izadi E 2013 Catal. Commun. 42104

26. Mistry S R, Joshi R S, Sahoo S K and Maheria K C 2011 Catal. Lett. 1411541

27. Rani V R, Srinivas N, Kishan M R, Kulkarni S J and Raghavan K V 2001 Green Chem. 3305

28. Kang L, Jin D and Cai Y 2013 Synth. Commun. 431896

29. Narahari S R, Reguri B R, Gudaparthi O and Mukkanti K 2012 Tetrahedron Lett. 531543 
30. Rajack A, Yuvaraju K, Praveen C and Murthy Y L N 2013 J. Mol. Catal. A 370197

31. Ahmed N and Siddiqui Z N 2014 J. Mol. Catal. A 387 45

32. Kolvari E, Koukabi N and Armandpour O 2014 Tetrahedron 701383

33. Yuan C, Huang Z and Chen J 2012 Catal. Commun. 24 56

34. Safari J and Zarnegar Z 2014 New J. Chem. 38358

35. Srivastava R 2010 Catal. Lett. 13917

36. Hallett J P and Welton T 2011 Chem. Rev. 1113508

37. Alvim H G O, de Lima T B, de Oliveira H C B, Gozzo F C, de Macedo J L, Abdelnur P V, Silva W A and Neto B A D 2013 ACS Catal. 31420

38. Dadhania A N, Patel V K and Raval D K 2012 J. Chem. Sci. 124921

39. Shaabani A and Rahmati A 2005 Catal. Lett. 100 177

40. Chavan S S, Sharma Y O and Degani M S 2009 Green Chem. Lett. Rev. 2175

41. Sharma N, Sharma U K, Kumar R and Richa Sinha A K 2012 RSC Adv. 210648

42. Hajipour A R, Khazdooz L and Zarei A 2011 Synth. Commun. $\mathbf{4 1} 2200$
43. Daily L A and Miller K M 2013 J. Org. Chem. 784196

44. Siddiqui I R, Srivastava A, Shamim S, Srivastava A, Waseem M A, Rahila S, Abumhdi A H, Srivastava A and Rai P 2014 J. Mol. Catal. A 382126

45. Roy S R, Jadhavar P S, Seth K, Sharma K K and Chakraborti A K 2011 Synthesis 142261

46. Elango K, Srirambalaji R and Anantharaman G 2007 Tetrahedron Lett. 489059

47. Nagarajan S and Elango K 2014 Catal. Lett. 1441507

48. Liu Q, Pan N, Xu J, Zhang W and Kong F 2013 Synth. Commun. 43139

49. Ramalingan C and Kwak Y 2008 Tetrahedron 645023

50. Heravi M M, Derikvand F and Bamoharram F F 2005 J. Mol. Catal. A 242173

51. Litvic M, Vecenaj I, Ladisic Z M, Lovric M, Vinkovic V and Litvic M F 2010 Tetrahedron 663463

52. Wanmei L 2011 Heterocycles 832067

53. Mukhopadhyay C and Datta A 2010 J. Heterocycl. Chem. 47136

54. Pore M D 2007 Aust. J. Chem. 60435

55. Misra A K, Agnihotri G and Madhusudan S K 2004 Indian J. Chem. B $\mathbf{4 3} 2018$

56. Aridoss G and Jeong Y T 2010 Bull Korean Chem. Soc. 31863 